\title{
Consumers' ability to match foods to the eatwell plate
}

\author{
Q.Q. $\mathrm{Ni}^{1}$, S. Whybrow ${ }^{2}$ and L.C.A. Craig ${ }^{2}$ \\ ${ }^{1}$ Robert Gordon University, Aberdeen $A B 107 Q B$ and ${ }^{2}$ Rowett Institute of Nutrition and Health, University of \\ Aberdeen, Aberdeen AB25 2ZD
}

In the UK, the eatwell plate was developed to provide a consistent message to the public about how to achieve a healthy balanced diet $^{(1)}$. The eatwell plate is a food-based visual representation of the balance of five food groups: bread, rice, potatoes, pasta and other starchy foods (starchy), fruit and vegetables (F\&V), milk and dairy foods (dairy), meat, fish, eggs, beans and other non-dairy sources of protein (protein) and foods and drinks high in fat and/or sugar (HFHS) ${ }^{(1)}$. Manufactured and homemade dishes typically comprise ingredients from several food groups and consumers "... just need to work out the main ingredients and think about how these fit with the sections on the [eatwell] plate"(2). Consumers' ability to do this does not appear to have been evaluated despite the need for this being suggested previously ${ }^{(3)}$. This study aimed to address this.

Forty-four adults (aged 18-55, 17 F \& $27 \mathrm{M}$ ) were provided with published information on the eatwell plate (2), sequentially shown 12 photographs and descriptions of common foods and composite dishes of increasing complexity, and asked to estimate the eatwell plate proportions of each. Proportions of the five eatwell food groups were calculated by researches using published guideline methods ${ }^{(2)}$, with composite foods being disaggregated. Subjects' estimates were compared for agreement ( $\pm 25 \%$ to allow for ambiguity in the portion sizes) to the reference proportions, and the proportions compared using the Aitchison distance, which quantifies similarity of compositional data with zero being perfect agreement.

\begin{tabular}{|c|c|c|c|c|c|c|c|}
\hline Food item & Starchy $\%$ & $\mathrm{~F} \& \mathrm{~V} \%$ & Dairy \% & HFHS $\%$ & Protein $\%$ & Correct $n$ & Agreement Aitchison distance \\
\hline Greek yogurt & $3(0)$ & $2(0)$ & $76(100)$ & $14(0)$ & $4(0)$ & 26 & 3.9 \\
\hline Ice cream & $5(0)$ & $1(0)$ & $50(0)$ & $41(100)$ & $4(0)$ & 3 & $6 \cdot 2$ \\
\hline Black pudding & $10(0)$ & $1(0)$ & $2(0)$ & $24(0)$ & $64(100)$ & 18 & $5 \cdot 0$ \\
\hline Macaroni cheese & $43(39)$ & $1(0)$ & $31(58)$ & $17(3)$ & $8(0)$ & 12 & 3.9 \\
\hline Couscous with vegetables & $37(70)$ & $45(30)$ & $4(0)$ & $8(0)$ & $7(0)$ & 19 & $4 \cdot 8$ \\
\hline Cottage pie & $24(34)$ & $14(20)$ & $11(14)$ & $15(6)$ & $35(26)$ & 37 & $2 \cdot 3$ \\
\hline Ham, cheese \& salad sandwich & $31(30)$ & $15(20)$ & $15(10)$ & $15(10)$ & $25(30)$ & 37 & $2 \cdot 1$ \\
\hline Beef lasagne & $21(24)$ & $8(24)$ & $18(24)$ & $17(4)$ & $36(24)$ & 28 & $3 \cdot 0$ \\
\hline Prawn noodles & $32(35)$ & $20(33)$ & $4(0)$ & $10(0)$ & $34(32)$ & 33 & $4 \cdot 7$ \\
\hline Hawaiian pizza & $34(42)$ & $10(27)$ & $15(17)$ & $20(2)$ & $21(11)$ & 26 & $3 \cdot 3$ \\
\hline Fish \& chips & $29(0)$ & $4(0)$ & $3(0)$ & $27(75)$ & $37(25)$ & 3 & $6 \cdot 0$ \\
\hline Full English breakfast & $22(17)$ & $13(33)$ & $7(0)$ & $22(0)$ & $36(50)$ & 22 & $5 \cdot 8$ \\
\hline All the above & $24(25)$ & $11(15)$ & $19(17)$ & $19(15)$ & $28(27)$ & & \\
\hline
\end{tabular}

Correct, number of subjects estimating all five eatwell proportions within $25 \%$ of the reference proportions.

Surprisingly, agreement tended to be better for the composite foods than for those belonging to just one eatwell group. Difficulties appeared to be with foods where the correct eatwell group was not immediately obvious; chips being allocated to the starchy group rather than HFHS, ice cream to dairy rather than HFHS, and black pudding to HFHS rather than the protein foods group. Overall the estimated eatwell plate proportions of $50 \%$ of the foods were within $25 \%$ of the reference proportions.

This study suggests that consumers can identify the main food items in combination foods and fit them to the eatwell plate reasonably well, although the proportions of F\&V tended to be underestimated. Subjects appeared to have greater difficulty assigning the proportions of foods to the HFHS eatwell plate food group, suggesting that, for certain foods, this part of the eatwell plate message is unclear.

1. Food Standards Agency (2010) The eatwell plate.

2. Public Health England (2014) Your guide to eatwell plate: helping you eat a healthier diet.

3. Gatenby SJ et al. (1995) The National Food Guide: development of dietetic criteria and nutritional characteristics. J Hum Nutr Diet 8, 323-334. 